\title{
Predicting the tensile properties of additively manufactured Ti-6Al-4V via electron beam deposition
}

\author{
Thomas Ales $^{1}$, Iman Ghamarian ${ }^{1}$, Brian Hayes ${ }^{1}$, Brian Welk ${ }^{2}$, Andrew Baker ${ }^{3}$, Matthew Kenney ${ }^{1}$, D Gary Harlow ${ }^{4}$, Hamish Fraser ${ }^{2}$, \\ Wenqi $\mathrm{Li}^{5}$, Peter Collins ${ }^{*}, 1$
}

1: lowa State University, Ames, IA, United States

2: Ohio State University, Columbus, $\mathrm{OH}$, United States

3: The Boeing Company, St. Louis, MO, United States

4: Lehigh University, Bethlehem, PA, United States

5: University of Nottingham, Nottingham, United Kingdom

\begin{abstract}
Additively manufactured materials are gaining wide attention owing to the manufacturing benefits as it results in near net shape components. It is well known that the manufacturing processes affects the performance of the components via microstructural features and the mechanical properties. There is an urgent need to understand the processing-structure-property-performance relationship for the materials manufactures via such innovative techniques. Strategies are needed to quantify and modify the mechanical properties. This study assists to design and tailor the process parameters based on the final properties required. Current work predicts the yield strength of additively manufactured Ti-6Al-4V with different post heat treatments. A thermal model predicted by ABAQUS is fed into an implementation of Langmuir equation that predicts the chemistry which is then used in a phenomenological equation predicting the yield strength. The model is confirmed via experiments showing less than $2 \%$ deviation from the predicated properties. A statistical model gives design allowables that have an uncertainty of less than $1 \mathrm{ksi}$.
\end{abstract}

\section{Introduction}

In the main, it is desirable to have an integrated computational materials engineering (ICME) strategy to predict the properties of additively manufactured materials, and thereby accelerate the application of these advanced manufacturing approaches by various commercial companies. In any ICME strategy, it is necessary to fully understand and capture the relevant details of the materials science paradigm: composition, processing, microstructure, properties, and performance.

This work represents a multi-institution, 6-year effort to develop a robust ICME strategy capable of predicting the properties and performance of additively manufactured Ti-6Al-4V to within $7 \mathrm{MPa}(1 \mathrm{ksi})$. Key components of the framework include: (i) a thermal model; (ii) a Langmuir-based model to predict the asdeposited composition; (iii) a phase field model to predict the microstructure; (iv) an artificial neural network-genetic algorithm extracted phenomenological equation to predict strength; $(v)$ a statistically based model for the probabilistic prediction of strength; and (vi) an ABAQUS model and framework that integrates the knowledge of the program into a predictive tool.

\section{Materials and Experiments}

(C) The Authors, published by EDP Sciences. This is an open access article distributed under the terms of the Creative Commons Attribution License 4.0 (http://creativecommons.org/licenses/by/4.0/). 
To develop an ICME framework, a large-scale method of additive manufacturing (i.e., the Sciaky electron beam additively manufactured (EBAM) process) was selected to produce Ti-6Al-4V. This particular method uses a high energy electron beam as a heat source, which operates under vacuum, and feeds in a wire that is melted and added to the previously deposited material. The as-deposited material was sectioned and subjected to various thermal histories [1]. The static mechanical properties were determined, and a database was developed that consisted of measurements of the as-deposited composition, microstructure, and mechanical properties. A variety of state-of-the-art materials characterization techniques were adopted, ranging from the more routine backscattered electron microscope images that were subsequently quantified to advanced precession electron diffraction techniques that were applied to spatially resolve and quantify dislocation densities by relating the presence of such defects to lattice curvature at a nm length scale [2].

Following materials characterization, a physically based equation was developed using a hybrid artificial neural network - genetic algorithm approach that has been described elsewhere. Briefly, this approach permits a genetic algorithm approach to match an n-variable equation that has been determined using the flexible artificial neural network to a n-variable phenomenological equation that has been postulated based upon known physics. The resulting equation overcomes several of the limitations of artificial neural networks, including deviations in the equation in regions of data sparsity. This equation, originally developed for wrought Ti-6Al-4V, was modified slightly for the additively manufactured material. Specifically, a texture debit term was introduced for the observed decrease in yield strength along the primary $x-y-z$ cartesian coordinate axis, and a Taylor hardening terms incorporated for variations in dislocation density.

A sophisticated statistical approach was incorporated to manage the small variability that exists between the predicted values and the experimentally measured values when both simulated and experimentally measured data is plotted on a cumulative probability distribution function plot (c-pdf). A statistical adjustment of the model data and experimental data is sufficient to apply to future data and have such future data lie almost perfectly on the c-pdf.

In parallel, an ABAQUS code was developed to predict: the thermal history; the chemistry variation due to volatilization of certain species under vacuum; the microstructure; and the resulting static mechanical properties. The predicted c-pdfs were compared to the measured c-pdfs and found to be in very good agreement.

\section{Results and Discussion}

The results of this work are extensive, and too broad to appear in this proceedings paper. For complete descriptions, the reader is pointed elsewhere [3-6]. The most important details of this work are given below.

\section{The Equation:}

The phenomenological equation that permits the prediction of the yield strength of Ti-6Al-4V is given below. The equation is built upon the summation of the individual strength contributions, starting with the average intrinsic flow stresses of the hcpalpha and bccbeta phases, followed by compositional increases to strength (i.e., solid solution and interstitial strengthening from Al, V, O, and Fe), followed by Hall-Petch related terms, a modification due to texture, and Taylor hardening. This equation has been used to predict accurately the strength of wrought and additively manufactured Ti-6Al-4V, with variations in chemistry, including custom variations beyond the form of the equation presented here. The variables are phase fraction, morphological dimensions, and compositional terms [5]. 


$$
\sigma_{y s}=\left\{\begin{array}{l}
F_{V}^{\alpha} \cdot 89+F_{V}^{\beta} \cdot 45+ \\
F_{V}^{\alpha} \cdot\left(149 \cdot x_{A l}^{0.667}+759 \cdot x_{O}^{0.667}\right)+F_{V}^{\beta} \cdot\left(\left(22 \cdot x_{V}^{0.7}\right)^{0.5}+\left(235 \cdot x_{F e}^{0.7}\right)^{0.5}\right)^{2}+ \\
F_{V}^{c o l} \cdot 150 \cdot\left(t_{\alpha-l a t h}\right)^{-0.5} \cdot\left(t_{\beta-r i b}\right)^{0.5}+ \\
F_{V}^{c o l} \cdot 125 \cdot\left(t_{\text {colony }}\right)^{-0.5}+ \\
(-1) \cdot(\text { AxisDebit })+ \\
F_{V}^{B W} \cdot \alpha M G b \sqrt{\rho}
\end{array}\right.
$$

\section{Compositional Variations:}

In additive manufacturing, the molten pool can be quite hot. When this pool is present under an inert gas, it is possible that the molten pool will getter trace amounts of elements in the inert gas (i.e., $\mathrm{O}, \mathrm{N}, \mathrm{H}$ ). When this pool is under vacuum, certain alloying species will be preferentially lost to the vacuum. For Ti-6Al-4V, this preferential volatilization reduces the concentration of aluminum. A reasonable model to predict the loss of aluminum (or the uptake of $\mathrm{O}, \mathrm{N}, \mathrm{H}$ under positive pressures) is based upon a model proposed by Langmuir, which predicts the mass loss across a given interface, and which has been adopted elsewhere [79]. In this model, it is important to know the temperature of the molten pool, the time a given surface will be available to mediate mass flux, and the area of the molten pool. Interestingly, for additively manufactured materials, the calculation of solute profiles in the liquid pool using, for example, Fick's Second Law, are not necessary, as the convective motion of the liquid is quite high ( 0.5 to $1.0 \mathrm{~m} / \mathrm{s})$, causing the solute to be actively redistributed in the liquid state, and thus enabling a simple mass-loss model. The simple Langmuir equation is given below:

$$
m=\sqrt{\frac{M_{i}}{2 \pi R T}} \cdot p_{i}
$$

where $m$ is the mass of atoms entering or exiting a surface, $M_{i}$ is the molecular weight, and $p_{i}$ is the partial pressure of species $i$.

\section{Microstructural Variation and Effects of Texture:}

During solidification under vacuum, the size of the molten pool ( 10 to $15 \mathrm{~mm}$ in diameter) is sufficiently large so as to promote multiple competing solidification fronts, especially in thick sections. These multiple solidification fronts lead to a "bamboo" like appearance (see Fig. 1), where some grains are epitaxially grown from an underlying layer, and adjacent grains are nucleated from the adjacent AM passes side surface. This results in a microstructures variation that is observable at the mesoscale $(\sim 1 \mathrm{~cm}$ frequency) but not at the microscale. This feature may be associated with signal complications when such specimens are subjected to nondestructive testing such as ultrasonic inspection. Subscale test coupons were extracted from the different zones of this periodic bamboo structure. The tests of the vertically banded regions resulted in mechanical properties that had very little scatter, whereas the vertical bands consisting of horizontally growing grains have a more random grain structure, and the corresponding mechanical properties are more broadly scattered.

The appearance of this particular feature drove the team to find alternative methods of materials characterization at the mesoscale. The authors are working with researchers at the University of Nottingham (U.K.) to apply a new method of orientation microscopy - spatially resolved acoustic spectroscopy (SRAS)- to these complicated structures. SRAS uses laser-induced surface acoustic waves and a relatively complex forward model to determine the local crystal orientation. The maps that are achievable using SRAS are quite large, with demonstrations of analysis over 20,000 square millimeters. One sub scale SRAS dataset is shown in Fig. 2(a), which clearly shows the banding structure. A large area run is shown in Fig. 2(b). 
The strong $\{001\}$ beta growth texture, along with the concomitant alpha variant selection, results in the softest crystallographic directions lying on planes oriented $45^{\circ}$ away from the primary cartesian coordinate axes $(x, y, z)$. A test of 36 coupons ( 6 for each of the primary axes $(x, y, z)$ and 6 for each $45^{\circ}$ off-axis test ( $x y$, $y z, x z)$ ) clearly demonstrated that the tests taken along the primary axes exhibit yield strengths $\sim 5.5 \%$ lower than the $45^{\circ}$ counterparts [5].

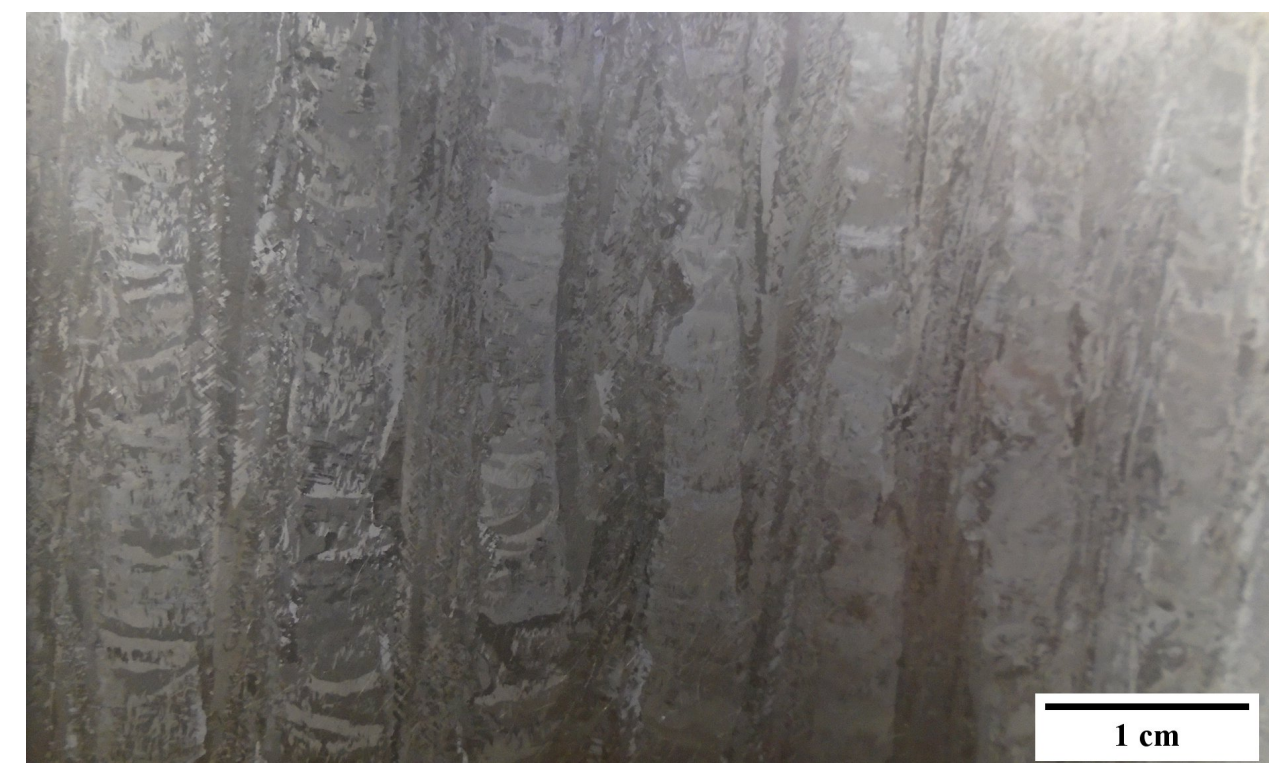

Figure 1: Bamboo structure in EBAM Ti-6Al-4V
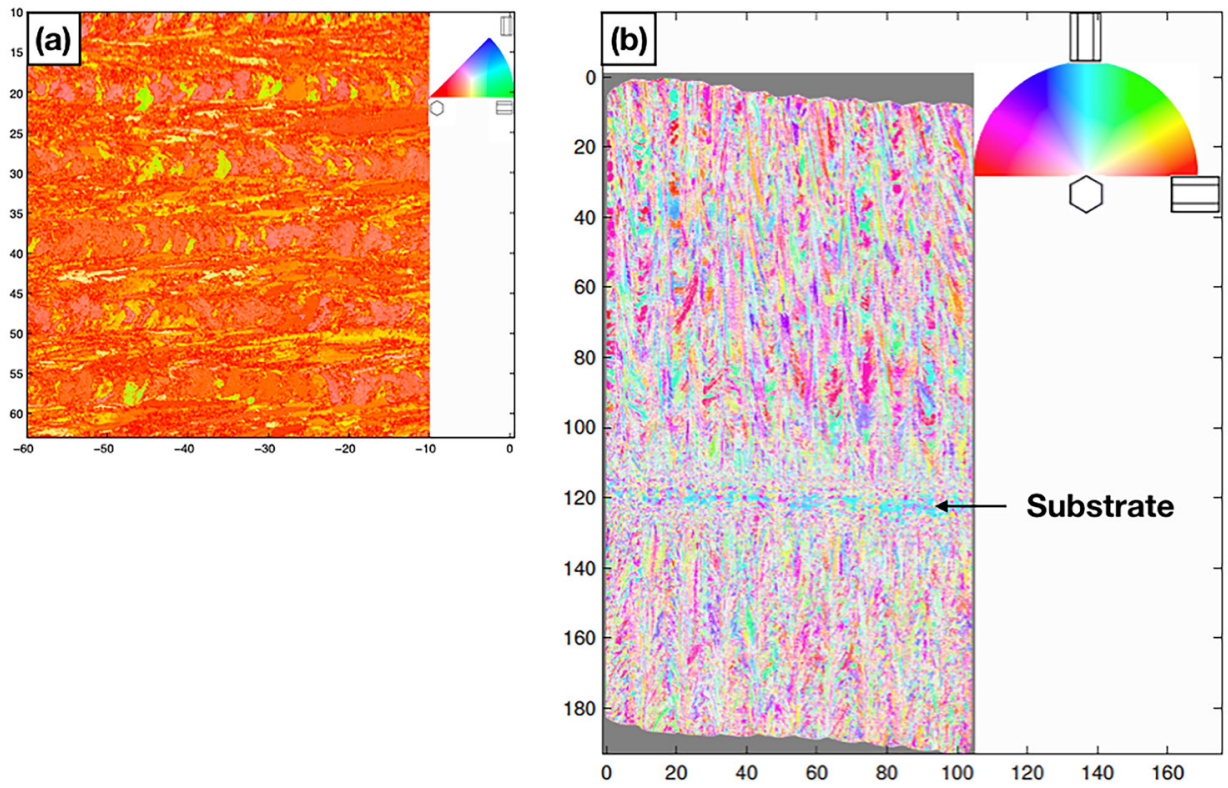

Figure 2: Results of SRAS (a) High resolution 50mmx50mm map. (b) lower resolution map of an entire AM cross-section.

\section{Dislocation Densities:}

One important contribution to strength in additively manufactured materials is Taylor Hardening. The dislocation densities have been measured for different material thermal treatments following deposition, and ranges from relatively low values $\left(\sim 10^{12} \mathrm{~m}^{-2}\right)$ to relatively high values $\left(\sim 10^{15} \mathrm{~m}^{-2}\right)$. This difference leads 
to a variation in the yield strength of the material, as well as a slight reduction in ductility. An image showing a PED map and the corresponding dislocation density map for the stress relieved material is shown in Fig. 3. We note, rather importantly, that residual stress and average dislocation density are two different measurements. Residual stress is considered to be the spatial gradient in dislocation density. Thus, it is possible to have a high average dislocation density, but a low residual stress if the gradient is low. This is an important consideration for additively manufactured materials. We note that, currently, we do not have definitive proof on the origins of such high dislocation densities, but are pursuing a few theories.

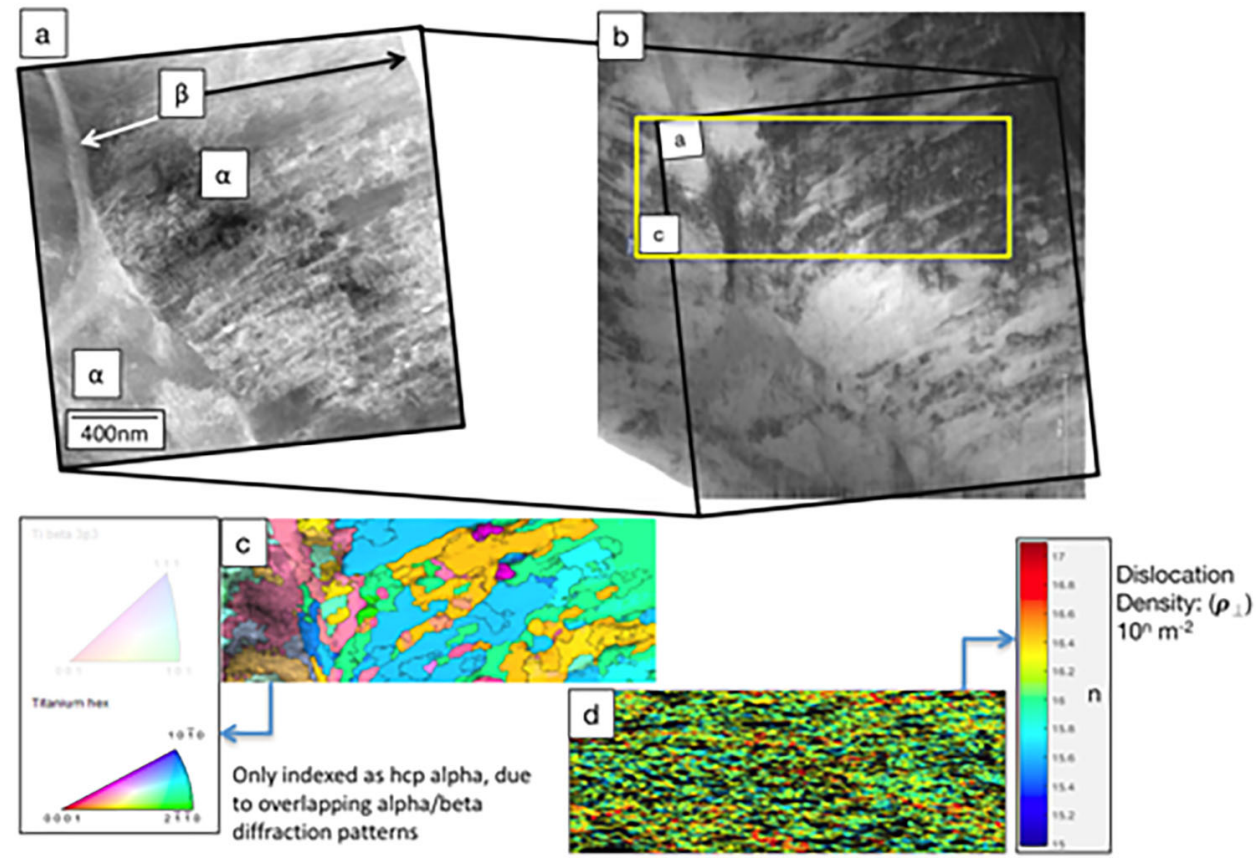

Figure 3: PED and GND map [5]

\section{Integrated Model:}

An ABAQUS model was developed that simulated the EBAM process, and integrated the multiple levels of physics that was required to predict the properties and performance. In addition to predicting composition, microstructure was predicted using two approaches, namely a phase field model and a database strategy. Similarly, the dislocation density was incorporated using a database approach. Following the linkage of these databases and the ABAQUS model, a series of "virtual tensile" coupons were made on the virtual depositions, and the populations compared with what was experimentally measured. The results are in excellent agreement, and shown in Fig. 4. 


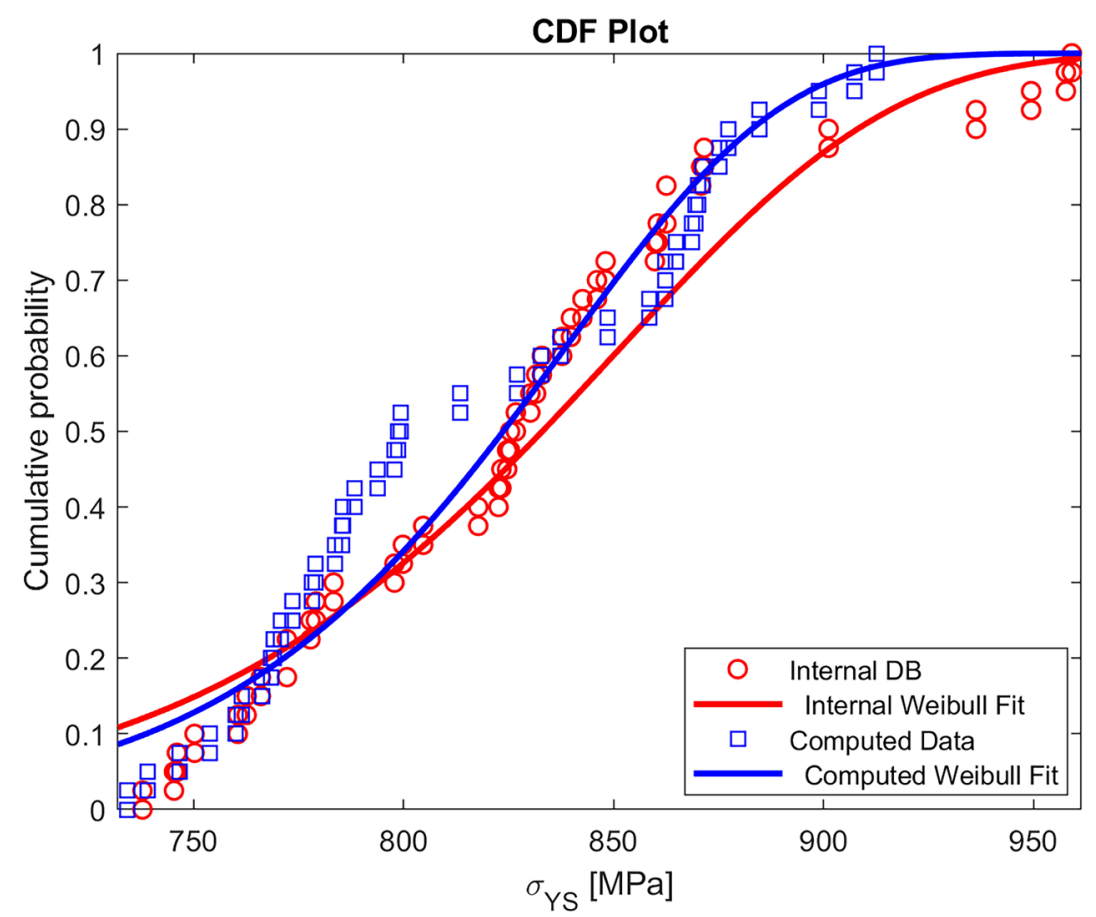

Figure 4: ABAQUS model and simulated data, compared with measured. This data represents a limited number of data, and large datasets, once tuned, are indistinguishable from experimentally measured values.

\section{Conclusion}

An ICME framework has been established to predict the static strength properties and their cumulative probabilities to predict performance for electron beam additively manufactured Ti-6Al-4V. Important factors in the strength model include composition, texture, dislocation density, and the microstructures features. Once developed, the models are able to predict the tensile strength within $\sim 1 \mathrm{ksi}(\sim 7 \mathrm{MPa})$.

\section{Acknowledgements}

The authors acknowledge the support of the DARPA contract HR0011-12-C-0035 ("An Open Manufacturing Environment for Titanium Fabrication") and the Al and Julie Renken Professorship. The authors also gratefully acknowledge the help of Lindsay Winters and Scott Wells for their help on some supporting characterization. The views, opinions and/or findings expressed are those of the author and should not be interpreted as representing the official views or policies of the Department of Defense or the U.S. Government.

\section{$\underline{\text { References }}$}

1. A.H. Baker, P.C. Collins, J.C. Williams, JOM. 69 (7) (2017) 1221-1227.

2. I. Ghamarian, P. Samimi, V. Dixit, P.C. Collins, Metall Mater Trans A. 46 (11) (2015) 5021-5037.

3. P.C. Collins, C.V. Haden, I. Ghamarian, B.J. Hayes, T. Ales, G. Penso, V. Dixit, G. Harlow, JOM. 66 (7) (2014) 1299-1309.

4. C.V. Haden, P.C. Collins, D.G. Harlow, JOM. 67 (6) (2015) 1357-1361.

5. B.J. Hayes, B.W. Martin, B. Welk, S.J. Kuhr, T.K. Ales, D.A. Brice, I. Ghamarian A.H. Baker, C.V. Haden, D.G. Harlow, H.L. Fraser, P.C. Collins, Acta Mater. 133 (2017) 120-133.

6. B. Hayes, I. Ghamarian, S. Joshi, R. Banerjee, N. Dahotre, P. Collins, V. Dixit, B. Welk, H. Fraser, SAMPE. 2015.

7. I. Langmuir, Phys rev. 2 (5) (1913) 329.

8. S.L. Semiatin, V.G. Ivanchenko, O.M. Ivasishin, Metall Mater Trans B. 35 (2) (2004) 235-245.

9. P.C. Collins, D.A. Brice, P. Samimi, I. Ghamarian, H.L. Fraser, Ann Rev Mater Res. 46 (2016) 63-91. 